\title{
A CONVERGENCE PROBLEM FOR CONTINUED FRACTIONS ${ }^{1}$
}

\author{
T. L. HAYDEN
}

In this paper a sequence $V$ of regions in the complex plane is called an admissible sequence provided that:

(i) for $n \geqq 2, V_{n}$ is either a circle with center the origin plus its interior $\left(C_{0}+\right.$ int.), or a circle with center the origin plus its exterior ( $C_{0}+$ ext. $)$, and

(ii) the continued fraction

$$
1 / 1+{ }^{a_{2} / 1}+{ }^{a_{3} / 1}+{ }^{a_{4} / 1}+\cdots
$$

converges if for $n \geqq 2, a_{n} \in V_{n}$.

The problem that is raised in this paper, and to which the following theorems contribute a partial solution, is the problem of finding all admissible sequences for (1.1). The collection of all admissible sequences is denoted by AS.

Before stating the theorems, it is convenient to have some additional notation and definitions. The continued fraction (1.1) is considered as being generated by the sequence $t$ of linear fractional transformations defined by:

$$
t_{1}(z)=1 / z, \quad t_{n}(z)=1+a_{n} / z \quad n \geqq 2 .
$$

The sequence $T$ of linear fractional transformations is defined by:

$$
T_{1}(z)=t_{1}(z), \quad T_{n}(z)=T_{n-1}\left(t_{n}(z)\right) \quad n \geqq 2,
$$

so that

$$
T_{n}(z)=\frac{A_{n-1} z+a_{n} A_{n-2}}{B_{n-1} z+a_{n} B_{n-2}}
$$

where $a_{1}=1$ and

$$
\begin{array}{ll}
A_{0}=0, A_{1}=1 ; & A_{n}=A_{n-1}+a_{n} A_{n-2} ; \\
B_{0}=1, B_{1}=1 ; & B_{n}=B_{n-1}+a_{n} B_{n-2} .
\end{array}
$$

The $n$th approximant $f_{n}$ of $(1.1)$ is $T_{n}(1)=A_{n} / B_{n}$ for $n \geqq 1$.

THEOREM 1. Suppose $V$ is a sequence such that for each integer $n>1$

Presented to the Society, January 25, 1961 under the title Some convergence regions for continued fractions; received by the editors August 2, 1961 and, in revised form, April 11, 1962.

1 This paper is based on the author's doctoral dissertation written under the supervision of Professor H. S. Wall of the University of Texas. 
either $V_{n}$ is a $C_{0}+$ int. or $V_{n}$ is a $C_{0}+$ ext., and there exists an integer $p>1$ such that $V_{p}$ and $V_{p+1}$ are not bounded; then there exists a sequence $a$ of complex numbers such that $a_{n} \in V_{n}$ for $n>1$ and the continued fraction (1.1) diverges.

The proof of Theorem 1 is simplified by the following lemma.

Lemma 1. If each of $R$ and $S$ is a positive number, $u$ is a complex number not 1 , and $v$ is a complex number not 0 , then there exists a complex number $a$ and a complex number $b$ such that $|a|>R,|b|>S$ and $u=1+a /(1+b / v)$.

Proof. Let $n$ be a positive number such that $n|u-1|>R$ and $(n-1)|v|>S$. Then, if $a=n(u-1)$ and $b=(n-1) v$ the conclusion of Lemma 1 follows.

Proof of Theorem 1. Suppose $p$ is an integer such that $V_{p}$ and $V_{p+1}$ are not bounded. If the continued fraction

$$
1 / 1+{ }^{a_{p+2} / 1}+{ }^{a_{p+3} / 1}+\cdots
$$

diverges in the sense that the approximants have more than one limit point, then (1.1) diverges. Therefore, we assume that (1.7) does not diverge in the above mentioned sense and let $a_{p+2}$ be a complex number in $V_{p+2}$ so that (1.7) converges to a complex number $v$ not 0 . If $p=2$ there exists, by Lemma 1 , an $a_{2}$ in $V_{2}$ and an $a_{3}$ in $V_{3}$ such that $0=1+a_{2} /\left(1+a_{3} / v\right)$. If $p>2$, let $a_{2}, a_{3}, a_{4}, \cdots, a_{p-1}$ be nonzero complex numbers in $V_{2}, V_{3}, V_{4}, \cdots, V_{p-1}$, respectively, such that $B_{p-2}$ and $B_{p-3}$ are not 0 and $-a_{p-1} B_{p-3} / B_{p-2}$ is not 1 . By Lemma 1 , there exists an $a_{p}$ in $V_{p}$ and an $a_{p+1}$ in $V_{p+1}$ such that, $-a_{p-1} B_{p-3} / B_{p-2}$ $=1+a_{p} /\left(1+a_{p+1} / v\right)$. We now note that by (1.3) and (1.4) $T_{1}(0)=\infty$ and $T_{p-1}\left(-a_{p-1} B_{p-3} / B_{p-2}\right)=\infty$ for $p>2$. Hence, the approximants $f_{n} \rightarrow \infty$ as $n \rightarrow \infty$.

REMARK 1. Lane and Wall [3] completely settled the problem of finding all admissible sequences where each region of the sequence is bounded by showing that, for $V \in A S$ (and $V_{n}$ bounded), it is necessary and sufficient that there exist a sequence $g$ of positive numbers less than 1 such that $V_{n}:|z| \leqq\left(1-g_{n-1}\right) g_{n}$.

TheOREM 2. Suppose $V$ is a sequence such that for $n \geqq 2$

(i) Either $V_{n}$ is a $C_{0}+$ int. or $V_{n}$ is a $C_{0}+$ ext.,

(ii) At least one of $V_{n}$ or $V_{n+1}$ is a $C_{0}+$ int., and

(iii) There exists a number $g_{n-1}$ and a number $r_{n}$ such that,

(a) $0<g_{n-1}<1,0<r_{n} \leqq 1$,

$$
V_{n}: \begin{cases}|z| \leqq r_{n}\left(1-g_{n-1}\right) g_{n} & \text { if } V_{n} \text { is bounded, } \\ |z| \geqq\left(1+g_{n-1}\right)\left(2-g_{n}\right) & \text { if } V_{n} \text { is not bounded, and }\end{cases}
$$


(b) if $p$ is an integer such that $V_{p+1}$ is not bounded, and $M$ is the collection of all such integers, then either $M$ is finite or $\prod_{k \text { in } M} r_{k}=0$. Then $V \in A S$.

Lemma 2.1. Suppose that $V$ is a sequence which satisfies the hypothesis of Theorem 2, $a_{n} \in V_{n}$, and for $n \geqq 1$,

$$
Z_{n}:\left\{\begin{aligned}
|z-1| & \leqq 1-g_{n} & & \text { if } V_{n+1} \text { is bounded, } \\
|z| & \geqq g_{n} & & \text { if } V_{n+1} \text { is not bounded }
\end{aligned}\right.
$$

then $t_{n}\left(Z_{n}\right) \subset Z_{n-1}$ for $n \geqq 2$.

Proof. For convenience the proof is divided into two cases: $V_{n}$ bounded and $V_{n}$ not bounded.

Suppose $V_{n}$ is bounded; consequently $Z_{n-1}:|z-1| \leqq 1-g_{n-1}$. If $z \in Z_{n}$ the minimum value of $|z|$ (for $V_{n+1}$ bounded or not bounded) is $g_{n}$. It follows that for $z$ in $Z_{n}$ and $a_{n}$ in $V_{n}\left|t_{n}(z)-1\right|=\left|a_{n}\right| /|z|$ $\leqq r_{n}\left(1-g_{n-1}\right) g_{n} / g_{n} \leqq 1-g_{n-1}$. Hence, $t_{n}\left(Z_{n}\right) \subset Z_{n-1}$ in case $V_{n}$ is bounded.

In case $V_{n}$ is not bounded, so that $V_{n+1}$ is bounded, then $Z_{n-1}:|z|$ $\geqq g_{n-1}$ and $Z_{n}:|z-1| \leqq 1-g_{n}$. The maximum value of $|z|$ for $z$ in $Z_{n}$ is $2-g_{n}$. Let $z \in Z_{n}$ and $a_{n} \in V_{n}$, then $\left|t_{n}(z)-1\right|$ $\geqq\left(1+g_{n-1}\right)\left(2-g_{n}\right) /\left(2-g_{n}\right)$; so that $\left|t_{n}(z)\right| \geqq g_{n-1}$, and hence the lemma is true for $V_{n}$ not bounded.

The following lemma constructs a sequence $U$ of circular regions each containing the next such that the $n$th approximant $f_{n}$ of (1.1) is in $U_{n}$.

LemMa 2.2. Suppose $V$ is a sequence which satisfies the hypothesis of Theorem 2, $a_{n} \in V_{n}, Z$ is a sequence which satisfies the hypothesis of Lemma 2.1, and $U$ is the sequence defined by: $U_{n}=T_{n}\left(Z_{n}\right)$ for $n \geqq 1$. Then, for $n \geqq 1, U_{n+1} \subset U_{n}$. Furthermore, $f_{n} \in U_{n}$.

Proof. We need only note, from Lemma 2.1 , that $t_{n}\left(Z_{n}\right) \subset Z_{n-1}$ and, by (1.3), $T_{n}(z)=T_{n-1}\left(t_{n}(z)\right)$; consequently, since $U_{n}=T_{n}\left(Z_{n}\right)$ $=T_{n-1}\left(t_{n}\left(Z_{n}\right)\right)$ and $U_{n-1}=T_{n-1}\left(Z_{n-1}\right)$, therefore $U_{n} \subset U_{n-1}$ for $n \geqq 2$. From the definition of $Z_{n}$ it is clear that $1 \in Z_{n}$, and since $T_{n}(1)=f_{n}$ it follows that $f_{n} \in U_{n}$.

Lемма 2.3. Suppose $V$ is a sequence which satisfies the hypothesis of Theorem 2 and $a_{n} \in V_{n}$. Then, for $p \geqq 2,\left|B_{p} / B_{p-1}-1\right| \leqq g_{p}$, if $V_{p}$ is bounded, and $\left|B_{p} / B_{p-1}-1\right| \geqq 2-g_{p}$, if $V_{p}$ is not bounded.

Proof. By (1.5), $B_{0}=1, B_{1}=1$, and $B_{p}=B_{p-1}+a_{p} B_{p-2}$, so that the lemma is true for $p=2$. Suppose the lemma is not true for some set $M$ of positive integers. Let $k+1$ be the least integer in $M$. Now by the 
assumption that $k+1$ is the least integer for which the inequalities fail, we see that $B_{k}$ and $B_{k-1}$ are not 0 , and hence by (1.5) $\left|B_{k+1} / B_{k}-1\right|$ $=\left|a_{k+1}\right| /\left|B_{k} / B_{k-1}\right|$.

We shall now consider two cases: $V_{k+1}$ bounded and $V_{k+1}$ not bounded. In case $V_{k+1}$ is bounded $\left|a_{k+1}\right| \leqq\left(1-g_{k}\right) g_{k+1}$. Also, $\left|B_{k} / B_{k-1}\right|$ $\geqq 1-g_{k}$, for $V_{k}$ bounded or unbounded. Hence $\left|B_{k+1} / B_{k}-1\right| \leqq g_{k+1}$.

In case $V_{k+1}$ is not bounded, $V_{k}$ is bounded, so that $\left|a_{k+1}\right|$ $\geqq\left(1+g_{k}\right)\left(2-g_{k+1}\right)$ and $\left|B_{k} / B_{k-1}\right| \leqq 1+g_{k}$. Hence $\left|B_{k+1} / B_{k}-1\right|$ $\geqq 2-g_{k+1}$ and the statement in the lemma is true for $k+1$. Consequently, the set $M$ does not exist.

We note that for $p \geqq 1, B_{p} \neq 0$ and $\left|B_{p}\right| \geqq\left|B_{p-1}\right|\left(1-g_{p}\right)$.

Proof of Theorem 2. In case $V_{n+1}$ is bounded for $n \geqq 1$ or $M$ is finite, then the proof follows from Remark 1 and Lemma 2.2. Suppose $p \in M, p>2$, and $M$ is infinite. Let $R_{p}$ and $C_{p}$ denote the radius and center respectively of $U_{p}$. Since $g_{p}$ is on the boundary of $Z_{p}$ and $T_{p}\left(Z_{p}\right)=U_{p}$, hence $R_{p}=\left|C_{p}-T_{p}\left(g_{p}\right)\right|$. Since points inverse in the circle $Z_{p}$ are mapped by $T_{p}(z)$ into points inverse in the circle $U_{p}$ it follows that $C_{p}=T_{p}\left(g_{p}^{2}\left[B_{p-1}\right]^{*} /\left[-a_{p} B_{p-2}\right]^{*}\right)$, inasmuch as $T_{p}\left(-a_{p} B_{p-2} / B_{p-1}\right)=\infty$. We may then use these expressions and (1.4) to show that

$$
R_{p}=\frac{\left|a_{p} g_{p}\right| \cdot\left|A_{p-1} B_{p-2}-A_{p-2} B_{p-1}\right|}{\left.\left|g_{p}^{2} \cdot\right| B_{p-1}\right|^{2}-\left|a_{p}\right|^{2} \cdot\left|B_{p-2}\right|^{2} \mid} .
$$

A similar argument, plus the use of (1.5), shows that

$$
R_{p-1}=\frac{\left|1-g_{p-1}\right| \cdot\left|A_{p-1} B_{p-2}-A_{p-2} B_{p-1}\right|}{\left.|| B_{p-1}\right|^{2}-\left(1-g_{p-1}\right)^{2} \cdot\left|B_{p-2}\right|^{2} \mid} .
$$

Since $\left|a_{p}\right| \leqq r_{p}\left(1-g_{p-1}\right) g_{p}$, Lemma 2.3 is sufficient to show that $R_{p} / R_{p-1} \leqq r_{p}$. By Lemma 2.2, $U_{p+1} \subset U_{p}$ and hence the sequence $R_{1}, R_{2}, R_{3}, \cdots$ is a nonincreasing sequence, consequently, the condition $\prod_{k \text { in } M} r_{k}=0$ is sufficient to show that the regions of the sequence $U$ have only one point in common, and hence (1.1) converges.

Theorem 3. Suppose $V$ is a sequence such that conditions (i), (ii), and (iii)-(a) of the hypothesis of Theorem 2 are satisfied with $r_{n}=1$ for $n \geqq 1$, and $\sum_{r=1}^{r=\infty} m_{1}, m_{2}, m_{3}, \cdots, m_{r}$ converges where

$$
m_{n}= \begin{cases}g_{n} /\left(1-g_{n}\right) & \text { if } V_{n} \text { is bounded, } \\ \left(2-g_{n}\right) /\left(1-g_{n}\right) & \text { if } V_{n} \text { is not bounded }\end{cases}
$$

then $V \in A S$. Moreover, the continued fraction (1.1) converges absolutely in this case.

PROOF. If some $a_{p}=0$, since $B_{p} \neq 0$, for $p \geqq 1$, then (1.1) converges. 
Hence we shall assume that $a_{p} \neq 0$ for $p \geqq 2$. Let

$$
h_{1}=0, \quad h_{p}=-a_{p} B_{p-2} / B_{p-1}
$$

then $T_{p}\left(h_{p}\right)=\infty$. Furthermore, since $T_{p}\left(h_{p}\right)=T_{p+1}\left(h_{p+1}\right)$ $=T_{p} t_{p+1}\left(h_{p+1}\right)$, then $h_{p}=t_{p+1}\left(h_{p+1}\right)$ which implies that $h_{p+1}$ $=-a_{p} /\left(1-h_{p}\right)$ for $p \geqq 1$. By (3.1) and Lemma 2.3, $\left|h_{p}\right| \leqq g_{p}$ if $V_{p}$ is bounded and $\left|h_{p}\right| \geqq 2-g_{p}$ if $V_{p}$ is not bounded. We now restate (1.4) in the following more useful form:

$$
T_{n}(z)=f_{n-1}+\left(a_{n} A_{n-2} B_{n-1}-a_{n} B_{n-2} A_{n-1}\right) / B_{n-1}^{2}\left(z-h_{n}\right) .
$$

Since $T_{n}(0)=f_{n-2}$ and $T_{n}(1)=f_{n}$ we see from (3.2) that

$$
\left|\left(f_{n}-f_{n-1}\right) /\left(f_{n-1}-f_{n-2}\right)\right|=\mid\left(h_{n} /\left(1-h_{n}\right) \mid\right. \text {. }
$$

Lane [2] proved that (1.1) converges absolutely, if there exists a sequence $d$ of positive numbers such that, $\left|h_{n} /\left(1-h_{n}\right)\right| \leqq d_{n} /\left(1+d_{n+1}\right)$ for $n \geqq 1$. Let $\mu_{n}=\left|h_{n} /\left(1-h_{n}\right)\right|$, then the sequence $d$ will exist provided $\sum_{r=1}^{r=\infty} \mu_{1} \mu_{2} \mu_{3} \cdots \mu_{r}$ converges. The maximum value of $\left|h_{n} /\left(1-h_{n}\right)\right|$ is $g_{n} /\left(1-g_{n}\right)$ if $V_{n}$ is bounded and it is $\left(2-g_{n}\right) /\left(1-g_{n}\right)$ if $V_{n}$ is not bounded. Hence the conditions of Theorem 3 are sufficient for the absolute convergence of (1.1).

Theorem 4. Suppose each of $s$ and $q$ is a positive number and $0<r<1$, and for $n \geqq 1,\left|a_{3 n-1}\right| \geqq(1+q+s)^{2},\left|a_{3 n}\right| \leqq r q$, and $\left|a_{3 n+1}\right| \leqq r s$. Then the sequence of approximants $f_{2}, f_{3}, f_{5}, f_{6}, \cdots, f_{3 n-1}, f_{3 n}, \cdots$ of (1.1) converges.

Lemma 4.1. Suppose $a_{2}, a_{3}, a_{4}, \cdots$ is a sequence which satisfies the hypothesis of Theorem 4 and for $n \geqq 1 ; Z_{3 n-2}:|z| \geqq s+q, Z_{3 n-1}:|z-1|$ $\leqq s+q$, and $Z_{3 n}:|z-1| \leqq s /(s+q)$. Then $t_{k}\left(Z_{k}\right) \subset Z_{k-1}$ for $k \geqq 2$.

A proof, similar to that of Lemma 2.1, is omitted. We note that 0 may be in $Z_{3 n-1}$, and in this case $t_{3 n-1}(0)=\infty$, a point in $Z_{3 n-2}$.

LEMMA 4.2. If (1) $a_{2}, a_{3}, a_{4}, \cdots$ is a sequence which satisfies the hypothesis of Theorem 4, (2) $Z_{1}, Z_{2}, Z_{3}, \cdots$ is a sequence which satisfies the hypothesis of Lemma 4.1, and (3) for $n \geqq 1, U_{n}=T_{n}\left(Z_{n}\right)$; then $U_{n+1} \subset U_{n}$.

A proof, similar to that of Lemma 2.2 is omitted.

We note that $f_{3 n} \in U_{3 n}$ and $f_{3 n-1} \in U_{3 n-1}$, but $f_{3 n+1}$ is not in $U_{3 n+1}$ in case $s+q>1$.

LEMMA 4.3. If $a_{2}, a_{3}, a_{4}, \cdots$ is a sequence which satisfies the hypothesis of Theorem 4 , then for $n \geqq 1$; (1) $\left|B_{3 n-1} / B_{3 n-2}-1\right| \geqq(1+q+s)$, (2) $\left|B_{3 n} / B_{3 n-1}-1\right| \leqq q /(q+s)$, and (3) $\left|B_{3 n+1} / B_{3 n}-1\right| \leqq q+s$. 
A proof, similar to that of Lemma 2.3, is omitted.

InDiCATION OF A PROOF OF THEOREM 4. Methods similar to those used in the proof of Theorem 2 may be used to show that, for $n \geqq 1$, $R_{3 n} / R_{3 n-1} \leqq r$, where $R_{n}$ is the radius of the region $U_{n}$. This is sufficient to show that the regions of the sequence $U$ have only one point in common.

We note that if $s+q \leqq 1$ in Theorem 4 , then $f_{n} \in U_{n}$ for every $n$, and hence (1.1) converges. This is a better result than can be obtained from Theorem 2, since the convergence of (1.1) can be obtained from Theorem 2 only in case $s+q<1$.

Corollary 4.1. Suppose $0<2 s<1,0<r<1$, and for each positive integer $n, V_{3 n-1}:|z| \geqq(1+2 s)^{2}, V_{3 n}:|z| \leqq r s$, and $V_{3 n+1}:|z| \leqq r s$. Then $V$ is in $A S$.

The regions in Corollary 4.1 are best in the sense that if $t>0$ and $0<c<4 t(1+t)$ then the periodic continued fraction such that, for $n \geqq 1, a_{3 n-1}=-(1+2 t)^{2}+c, a_{3 n}=a_{3 n+1}=t$ diverges.

Cowling, Leighton and Thron [1] proved that, for $n \geqq 1,\left|a_{2 n}\right| \leqq r^{2}$, $\left|a_{2 n+1}\right| \geqq 2\left(r^{2}-\cos \arg a_{2 n+1}\right)+\delta$ for $\delta>0$ and $r>1$ is sufficient for convergence of (1.1).

The following argument shows that in the case of triple regions convergence is not obtained without suitable restrictions on the radii of the bounded regions.

THEOREM 5. Suppose $s>0, V_{3 n-1}:|z| \geqq s, V_{3 n}:|z| \leqq 1$, and $V_{3 n+1}$ : $|z| \leqq 1$. Then there exists a sequence $a_{2}, a_{3}, a_{4}, \cdots$ such that, $a_{n} \in V_{n}$ for $n \geqq 2$, and (1.1) diverges.

LemMa 5.1. The continued fraction (1.1) diverges in case $a_{p} \neq 0$ for $p \geqq 2$, and the following series converges:

$$
\begin{aligned}
& \left|\left(1+a_{2}\right) / a_{2}\right|+\left|a_{2}\left(1+a_{4}\right) / a_{3}\right|+\left|a_{3}\left(1+a_{5}\right) / a_{2} a_{4} a_{5}\right| \\
& \quad+\left|a_{2} a_{4} a_{5}\left(1+a_{7}\right) / a_{3} a_{6}\right|+\left|a_{3} a_{6}\left(1+a_{8}\right) / a_{2} a_{4} a_{5} a_{7} a_{8}\right| \\
& \quad+\left|a_{2} a_{4} a_{5} a_{7} a_{8}\left(1+a_{10}\right) / a_{3} a_{6} a_{9}\right|+\cdots .
\end{aligned}
$$

For a proof see Wall [6].

Proof of Theorem 5. Suppose $t$ is a number greater than $s$ and 1 , and let $a_{3 n-1}=t$, and $a_{3 n}=a_{3 n+1}=-1$ for $n \geqq 1$. In this case the series (5.1) reduces to

$$
|t+1| \sum_{p=1}^{p=\infty}\left|1 / t^{p}\right|
$$

Since this series converges for $t>1$, by Lemma 5.1 the continued fraction (1.1) diverges. 


\section{REFERENCES}

1. V. F. Cowling, Walter Leighton and W. J. Thron, Twin convergence regions for continued fractions, Bull. Amer. Math. Soc. 50 (1944), 351-357.

2. R. E. Lane, Absolute convergence of continued fractions, Proc. Amer. Math. Soc. 3 (1952), 904-913.

3. R. E. Lane and H. S. Wall, Continued fractions with absolutely convergent even and odd parts, Trans. Amer. Math. Soc. 67 (1949), 368-380.

4. H. S. Wall, Some convergence problems for continued fractions, Amer. Math. Monthly 64 (1957), 95-103.

UNIVERSITY OF TEXAS AND

UNIVERSITY OF KENTUCKY

\section{NOTE ON A NONLINEAR EIGENVALUE PROBLEM}

\section{MARVIN SHINBROT ${ }^{1}$}

1. In the theory of hydrodynamic stability, eigenvalue problems of the form

$$
L u+\frac{1}{\lambda} M u=\lambda u
$$

arise $[1$, p. 430$]$. Here, $L$ and $M$ denote ordinary differential operators, the order of $L$ exceeds that of $M$, and the boundary conditions are such that $L$ is self-adjoint. One of the questions of interest is whether there exist eigenvalues of this problem and, if so, whether the corresponding eigenfunctions are complete.

Replacing $\lambda$ by $1 / \lambda$, it is easy to see that if $L^{-1}$ exists, (1.1) is equivalent to

$$
\lambda u=A u+\lambda^{2} B u,
$$

where $A=L^{-1}$ and $B=-L^{-1} M$ are compact, and $A$ is symmetric. In this note, we shall consider the question of the completeness of the eigenfunctions of the following generalization of (1.2):

$$
\lambda u=A u+\lambda^{\alpha} B_{\lambda} u,
$$

where $\alpha>1, A$ is compact and symmetric, and $B_{\lambda}$, which, as the notation indicates, may depend on $\lambda$, is merely bounded. More precise

Received by the editors May 11, 1962.

1 The work reported here was supported by the United States Air Force under Grant AF-AFOSR-62-136. 